
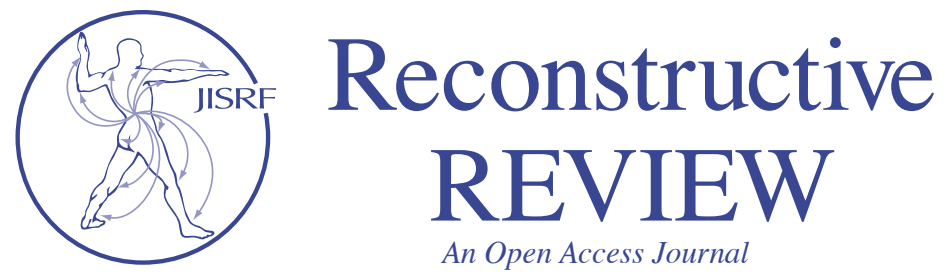

Check for updates

\title{
Early Term Radiographic Follow-Up of the Trident Tritanium Acetabular Component
}

DeBoer, $D^{1}$; Hodrick, $\mathrm{J}^{1}$; Christie, $M^{1}$

\section{Abstact}

Background: The purpose of this study was to evaluate the two year clinical and radiographic outcomes of patients undergoing a primary total hip arthroplasty (THA) using the Trident Tritanium primary Cup.

Methods: 197 patients who underwent a direct anterior THA using a Tritanium acetabular component between 2011 and 2015 were retrospectively reviewed by two surgeons from a single institution. The investigators, along with an independent physician, separately reviewed radiographs blinded to clinical data looking for radiolucent lines adjacent to the acetabular cup using the Charnley-DeLee zones. Clinical results were measured using acetabular revision surgery as an end point for failure. According to the American Academy of Orthopaedic Surgeons Levels of Evidence, this study was consistent with a Level III Therapeutic study.

Results: 101 (48.73\%) subjects did not exhibit any radiolucent lines around the acetabular component. 53 $(26.90 \%)$ subjects displayed radiolucency in only one zone. $27(13.71 \%)$ subjects displayed radiolucency in two zones, and $16(8.12 \%)$ displayed radiolucency in all three zones. Radiolucency was most prevalent in zone 1 at 2 years with $83(42.13 \%)$ subjects displaying radiolucency. There were seven $(2.54 \%)$ acetabular failures within two years of the index surgery. Of those 7 subjects, 3 displayed radiolucency in 1 zone, 2 displayed radiolucency in $>1$ zone, and 2 displayed radiolucencies $>1 \mathrm{~mm}$.

Conclusion: In our study, the Tritanium Cup demonstrated a $2.54 \%$ failure rate for aseptic loosening at 2 year follow-up. In addition, $51.27 \%$ of patients displayed a ra- diolucent line in at least one Charnley-DeLee zone. We also observed a progression of radiolucencies between the 6 month radiographs and the 2 year radiographs.

\section{Background}

The purpose of this review is to report a retrospective analysis of radiographic and clinical outcomes of a modern acetabular component with an ultra-porous biologic fixation surface. Specifically we are reporting to add to the body of literature regarding the Stryker Trident Tritanium (Stryker, Mahwah, NJ) acetabular component. There have been mixed reports on the short and midterm clinical and radiographic outcomes of patients undergoing total hip arthroplasty (THA) using the Trident Tritanium primary acetabular shell [1- $\underline{8}]$. In 2013, Naziri et al. reported on patients undergoing primary hip replacement surgery using the Tritanium cup and showed 100\% survival of the acetabular component at an average of 36 months follow-up [1]. A subsequent multicenter study with 12 centers and 255 cases demonstrated a $99.6 \%$ survivorship at 3-year follow-up [5]. Additionally, a six to ten year follow-up study of 62 patients undergoing revision hip arthroplasty using the Tritanium cup demonstrated $98 \%$ implant survivorship [2] . However, authors of more recent published studies voiced concerns regarding diminished implant lon-

Keywords: Primary Total Hip Arthroplasty; Biologic Fixation; Titanium; Acetabulum; Ultra Porous Surface; Treatment Outcome; Follow-Up Study; Radiography; Retrospective Study Level of Evidence: IV 
gevity and poor clinical outcome scores in primary THA $[\underline{9}, \underline{8}, \underline{3}]$. Carli et al. reported only two failures in 104 primary hips at a 5-year minimum follow-up study, but the radiographic analysis showed potentially pending failures with $40 \%$ of patients having radiosclerotic lines in 2 or more DeLee zones [ $\underline{3}$ ]. They also noted poorer functional outcomes in this same patient cohort, including lower postoperative Harris Hip Scores (HHS).

These concerns result from this acetabular shell being a member of a relatively new class of acetabular components that utilize an ultraporous metal substrate for the biologic fixation surface. Theoretically, these porous metal biologic fixation surfaces are designed to resemble trabecular bone and increase the rate of biologic fixation [10]. These surfaces can be manufactured to maintain an elastic modulus similar to trabecular bone and reduce stress shielding [11]. They also have a greater surface frictional coefficient to improve initial implant stability [12]. However, the long-term survivorship of implants manufactured with these newer technologies is unknown.

The purpose of this study is to report the radiographic results in primary THA of patients that received a Trident Tritanium acetabular component at a minimum of 2 year follow-up.

\section{Materials and Methods}

Between June 2012 and November 2015, we identified 200 consecutive patients that underwent primary total hip arthroplasty using a Tritanium acetabular component [13]. Three patients did not return for the minimum 2-year follow-up visit and were excluded from the review. This left 197 patients in the investigational cohort. This study received an Institutional Review Board (IRB) exemption letter for retrospectively reviewing a standard of care protocol for total joint arthroplasty. All patients included in the study met the normal criteria for primary total hip replace-

Table 1. Exclusion Criteria

\begin{tabular}{|l|}
\hline Criteria \\
\hline Age $<18$ yrs. old or $>79$ yrs. old \\
\hline BMI $>40 \mathrm{~kg} / \mathrm{m} 2$ \\
\hline Diagnosis of inflammatory arthropathy \\
\hline Prior organ transplant \\
\hline History of active joint sepsis \\
\hline Carcinoma within last 5 years (prior to surgery) \\
\hline Neurological disease (ex. Parkinson's disease) \\
\hline Psychosocial disorders \\
\hline Deficient bone stock requiring acetabular bone graft \\
\hline
\end{tabular}

ment and were in compliance with the labeling for the device. Exclusion criteria (Table 1) included age less than 18 years old or greater than 79 years old, a body mass index (BMI) greater than $40 \mathrm{~kg} / \mathrm{m} 2$, or a patient with a diagnosis of inflammatory arthropathy. Patients were also excluded if they had a prior organ transplant, history of active joint sepsis, carcinoma in the last 5 years, neurological disease (e.g. Parkinson's disease), psycho-social disorders that would limit rehabilitation, or deficient bone stock requiring use of a structural acetabular bone graft. Patient follow-up data was collected at 6 weeks, 3 to 6 months, 1 year, and minimum of 2 years post-operatively.

\section{Tritanium Acetabular Component}

All surgeries were performed using the Trident Tritanium Acetabular Cup System (Figure 1), which provides a variety of cup inserts and size options. The Tritanium components are cementless, made of titanium, available with a clusterhole pattern for adjunct fixation, and are available in sizes $48-80 \mathrm{~mm}$. The shells offer the option of Alumina Ceramic, Crossfire, or X3TM polyethylene inserts.

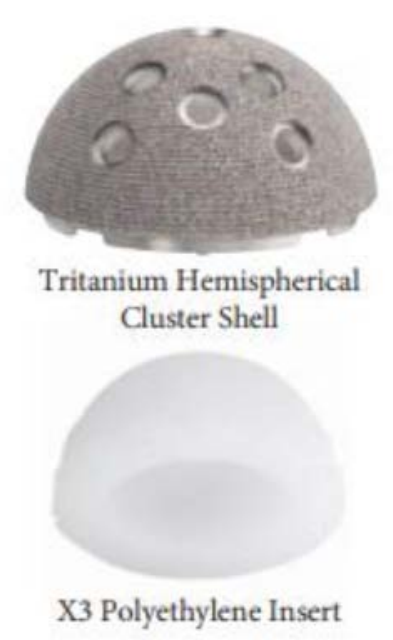

Figure 1. Tritanium Acetabular Cup Tritanium is the name of the surface coating on the underlying Trident shell. This surface manufacturing process begins with a mixture of a proprietary sacrificial pore former, polymeric binding agent, and commercially pure titanium powder [14]. The resulting material is molded and compacted at high pressure and low temperature to form a "green-state structure." The structure is machined to the desired form, mated to a titanium substrate, and treated to remove the pore former and binding agent. The process is completed by sintering the titanium particles to each other and the underlying substrate.

\section{Surgical Procedure}

All surgeries were performed by two fellowship trained surgeons (DKD, JTH) through a direct anterior approach using a Hana table. C-arm fluoroscopy was utilized in all procedures to aid with acetabular component positioning. Acetabular preparation was performed according to manufacturer protocol using the Stryker CuttingEdgeTM spherical reamers. Under-reaming the acetabulum by $1 \mathrm{~mm}$ was the preference for all cases; however, a few patients re- 
Table 2. Demographics

\begin{tabular}{|l|c|c|c|}
\hline & $\begin{array}{c}\text { Sample } \\
\text { Size (N) }\end{array}$ & $\begin{array}{c}\text { Mean } \\
\text { Value }\end{array}$ & $\begin{array}{c}\text { Standard } \\
\text { Deviation }\end{array}$ \\
\hline Age & 197 & 62.38 & 9.50 \\
\hline BMI & 197 & 28.49 & 4.70 \\
\hline Gender (M:F) & $(87: 110)$ & & \\
\hline Pre-Operative Diagnosis & & & \\
\hline Osteoarthritis & 175 & & \\
\hline Post-Traumatic Arthritis & 2 & & \\
\hline Avascular Necrosis & 20 & & \\
\hline
\end{tabular}

Table 3. Number of Patients with Radiolucencies at Various Time Intervals

\begin{tabular}{|l|c|c|c|}
\hline & 6 Months & 1 Year & 2 Years \\
\hline Zone 1 & 43 & 53 & 83 \\
\hline Zone 2 & 12 & 10 & 32 \\
\hline Zone 3 & 8 & 23 & 40 \\
\hline TOTAL & 51 & 59 & 96 \\
\hline & & & \\
\hline 1 Zone Affected & 41 & 36 & 53 \\
\hline 2 Zones Affected & 8 & 19 & 27 \\
\hline 3 Zones Affected & 2 & 4 & 16 \\
\hline
\end{tabular}

Table 4. Cup Failure Cases

\begin{tabular}{|c|c|c|c|c|c|c|c|c|}
\hline Patient & Age & BMI & $\begin{array}{c}\text { Failure Type or } \\
\text { Loosening }\end{array}$ & $\begin{array}{c}\text { Time to Failure } \\
\text { (months) }\end{array}$ & $\begin{array}{c}\text { 6 Month } \\
\text { Radiolucencies }\end{array}$ & $\begin{array}{c}\text { 1 Year } \\
\text { Radiolucencies }\end{array}$ & $\begin{array}{c}\text { 2 Year } \\
\text { Radiolucencies }\end{array}$ & $\begin{array}{c}\text { Latest Post-Op } \\
\text { Pain Score }\end{array}$ \\
\hline 1 & 46.8 & 32 & Aseptic & 51 & None & None & Zone 1,2,3 & 0 \\
\hline 2 & 66.1 & 36 & Aseptic & 18 & Zone 1 & Zone 1 & N/A & 3 \\
\hline 3 & 55 & 27 & Aseptic & 18 & None & None & N/A & 4 \\
\hline 4 & 77.1 & 30 & Aseptic & 7 & None & N/A & N/A & N/A \\
\hline 5 & 53.3 & 27 & Septic & 27 & None & Zone 1 & N/A & 7 \\
\hline 6 & 65 & 29 & Aseptic & 38 & None & None & Zone 1 & 5 \\
\hline 7 & 79 & 26 & Aseptic & 15 & Zone 1 & Zone 1,2 & N/A & 10 \\
\hline
\end{tabular}

ceived line to line reaming, at the surgeon's discretion, due to sclerotic bone. Cup positioning was determined by fluoroscopic images and reamer handle alignment rods with the goal of $40 \mathrm{o}$ of abduction and $20 \mathrm{o}$ of anteversion. One surgeon (DKD) preference was to use a single dome screw for adjunct acetabular fixation in all cases, while the other surgeon (JTH) assessed bone quality and used 0 , 1 , or 2 screws as he deemed necessary for initial stable fixation. Table 5 shows the breakdown in number of screws used in all cases. X3TM crosslinked polyethylene liners were used in all 197 cases. A 32 or 36mm ceramic femoral head was used in all cases. Patients with a $52 \mathrm{~mm}$ acetabular component or smaller received a $32 \mathrm{~mm}$ head ball. Patients with a $54 \mathrm{~mm}$ acetabular component or larger received a $36 \mathrm{~mm}$ head ball.

\section{Clinical Analysis}

Although the clinical outcomes were not primary focus of this review, patient variables such as age, sex, race, BMI, post-operative pain scores, and revision for aseptic loosening were recorded. In addition, intraoperative and post-operative complications were noted.

\section{Radiographic Analysis}

Radiographs taken at 6 weeks, 3 to 6 months, 1 year, and a minimum 2 year follow-up were analyzed by 3 orthopedic surgeons and were blinded to clinical outcome.
Table 5. Number of Cases with Adjuvant Screw Fixation

\begin{tabular}{|l|c|}
\hline & Number of Cases \\
\hline 0 Screws Used & 5 \\
\hline 1 Screw Used & 179 \\
\hline 2 Screws Used & 13 \\
\hline TOTAL & 197 \\
\hline
\end{tabular}

All patients received a standing anteroposterior pelvis $\mathrm{x}$ ray, anteroposterior hip, and lateral hip x-ray at each follow-up visit. Radiographs were inspected for the presence of radiolucent lines, the locations of which were documented using acetabular zones described by DeLee and Charnley [15]. Radiographs were also assessed for boneimplant gaps of $1 \mathrm{~mm}$ or greater. Three surgeons independently evaluated all radiographs and discrepancies in the presence or size of the radiolucency were resolved if 2 of 3 surgeons agreed. If no agreement in radiographic interpretation was reached, radiographs were re-evaluated sequentially at the available 6 week, 3 to 6 month, 1 year, and 2 year $\mathrm{x}$-rays to determine if a trend existed and consensus could be reached. Using this method, all discrepancies in radiographic findings were resolved for patients in this study. 


\section{Results}

There were 197 patients that underwent a primary total hip replacement using a Trident Tritanium acetabular component with adjuvant screw fixation and received radiographs at a minimum of 2 year follow-up. Patient demographics are shown in Table 2. There were 87 males and 110 females. The average age was $62.4+/-9.5$ years and the average BMI was $28.5+/-4.740 \mathrm{~kg} / \mathrm{m} 2$. Primary diagnoses included osteoarthritis in 175 patients, avascular necrosis in 20 patients, and post-traumatic arthritis in 2 patients. There were no significant intra-operative complications reported in this cohort.

There were 7 patients (Table 4) that were revised for acetabular loosening using revision surgery as the criteria for failure. Six patients (3.04\%) had aseptic implant loosening and 1 patient $(0.51 \%)$ experienced septic loosening; they had a history of alcoholism and developed stage 3 avascular necrosis. The patient underwent an uncomplicated primary total hip replacement but continued to report mild to moderate pain throughout the entire post-operative period. They have since had a progression of acetabular radiolucencies in 2 DeLee zones, increased uptake on Tc99 bone scan, and a hip aspiration that revealed greater than 100,000 WBCs. This patient ultimately grew Propionibacterium acnes from his hip aspirate, underwent a 2-stage revision for prosthetic joint infection, and has had no further sequalae related to his revision hip replacement.

In case 3 , the patient continued to have groin pain for almost the entire post-operative period (about 18 months). The patient was felt to have suspected iliopsoas impingement syndrome and surgical exploration of the hip demonstrated a loose acetabular component despite the lack of radiolucent lines. In case 4 , the patient had a posterior column fracture noted 6 weeks post-operatively from the index arthroplasty. A trial of restricted weight bearing was unsuccessful. A revision of the acetabular component with plating of posterior column was performed 7 months postoperatively. At the time of the revision surgery, the acetabular component was removed easily with no significant acetabular bone loss and sclerotic bone beneath the acetabular component.

The remaining six patients had isolated acetabular failures due to aseptic loosening. The average age of this group was $63.2+/-12.2$ years and the average BMI was $29.5+/-3.4 \mathrm{~kg} / \mathrm{m} 2$, which were not statistically different from our total cohort demographics. The average time to failure was 24.7 months. Four of these six patients did not have a radiolucent line at 6 months, but follow-up radiographs demonstrated a progression of their radiolucencies prior to implant failure. Two of the six patients had 1 zone of radiolucency at their 6-month visit. Of these two patients, one had a progression to 2 zones of radiolucency prior to failure; the other patient had a progression of 1 zone from 1 millimeter to 2 millimeters prior to failure. All six patients reported continued pain after surgery. The average Visual Analog Scale (VAS) Pain score for these six patients was 5.2 at final follow-up. Adjuvant screw fixation on the acetabular component was not standardized. Of the six aseptic failures, one patient had 2 screws, one patient had 0 screws, and the remaining four patients had 1 screw placed at the index surgery.

\section{Radiographic Analysis}

The number of patients with various radiolucencies at different time intervals are shown in Table 3. The total number of patients with radiolucent lines was 51 of 197 (26\%) at 6 months, 59 of $197(30 \%)$ at 1 year, and 96 of 197 (49\%) at 2 years. The majority of patients with radiolucencies were noted to be in zone 1 ; there were 43 patients with zone 1 radiolucencies noted at 6 months, 53 noted at 1 year, and 83 noted at 2 years. Zone 3 was the next most frequently noted location of radiolucency; there were 8 patients with zone 3 radiolucencies noted at 6 months, 23 noted at 1 year, and 40 noted at 2 years. Zone 2 radiolucencies were observed least frequently; there were 12 patients with zone 2 radiolucencies at 6 months, 10 noted at 1 year, and 32 noted at 2 years.

Radiolucent lines were observed in either one or multiple zones at the different time intervals. At 6 months, there were 41 patients $(21 \%)$ with 1 zone involved, 8 patients (4\%) with 2 zones involved, and 2 patients (1\%) with 3 zones involved. At 1 year, there were 36 patients (18\%) with 1 zone involved, 19 patients (10\%) with 2 zones involved, and 4 patients $(2 \%)$ with 3 zones involved. Finally, at 2 years, there were 53 patients $(27 \%)$ with 1 zone involved, and 27 patients (14\%) with 2 zones involved, and 16 patients $(8 \%)$ with 3 zones involved.

\section{Discussion}

In this study, the overall aseptic failure rate for isolated Tritanium acetabular implants at 2 years post-operatively was $3 \%$. These results are similar to those reported in national registries such as the Australian Joint Registry, which reported a $2.5 \%$ failure rate at 2 years, and the English National Registry, which reported a $1.63 \%$ failure rate at 3 years $[\underline{16}, \underline{17}]$. Furthermore, the aseptic loosening rates, using revision surgery as an endpoint for failure, reported on 1,038 primary THA patients with mid-term follow-up (24 - 72 months) were less than $2 \%$ for all studies 
using a Tritanium acetabular component []․

Concerns have also been raised about the number of patients with post-operative radiolucent lines around the acetabular component. Carli et al. were the first to report a concern regarding this issue in 2017 [ $\underline{3}$ ]. This study's 1 year radiographs demonstrated $30.3 \%$ of cases with radiolucent lines in 2 or more DeLee zones, of which $8.2 \%$ had radiolucent lines in 3 zones. During minimum 5 years followup, these numbers increased to $40.0 \%$ and $17.1 \%$, respectively. Additionally, Carli et al. compared the Tritanium cup patients to a cohort of patients receiving a conventional Trident hydroxyapatite (HA) coated acetabular implant [3]. The functional outcomes of the Tritanium cohort were statistically worse than the Trident HA group, suggesting impending failures in the Tritanium patients.

Yoshioka et al. also compared a group of Tritanium cup patients to a cohort of Trident HA cup patients []]. Each group contained 130 cases with an average follow-up slightly more than 3 years. The Tritanium cup group had at least 1 zone radiolucent line in $36 \%$ at 3 months and $60 \%$ at 3 years; in comparison, the Trident HA group experienced at least 1 zone of radiolucency in $2.5 \%$ at 3 months and in $0.8 \%$ at 3 years. They reported no difference in clinical outcome between groups at final follow-up of at least 3 years.

Another ultra-porous acetabular component that has been reviewed is the Dynasty Biofoam cup (MicroPort Orthopedics, Arlington, TN, USA). Carli et al. compared 92 patients (96 cups) implanted with the Biofoam cup to 93 patients (96 cups) using a traditional Trident peripheral self-locking (PSL) cup [18]. They also noted a large number of radiolucent lines on radiographs at a minimum follow-up of 2 years. A significantly greater number of Biofoam components exhibited radiolucencies in 2 zones (27.2\%) and 3 zones (12.0\%) compared to $0 \%$ of the Trident shells $(\mathrm{p}<0.05)$.

We also observed a significant number of patient radiographs with post-operative radiolucencies. In our study, at least 1 zone of radiolucency was detected in $26 \%$ of patients at 6 months, $30 \%$ at 1 year, and $49 \%$ at 2 years. Furthermore, the number of patients with radiolucencies increased over time. Our data showed that $21 \%$ of patients had 1-zone involvement on 6 month radiographs, which increased to $27 \%$ of patients at 2 years. Likewise, the number of patients with 2 or more zones of involvement increased over time. At 6 months, 2-zone of involvement was noted in $4 \%$ of patients and 3-zone involvement was noted in $1 \%$ of patients; these values increased to $14 \%$ and $8 \%$ at 2 years, respectively. This concerning number of radiolucencies warrants more frequent follow up for these patients. Our practice is to see all patients annually for the first 5 years.

There are several limitations to our study. The surgical technique was not standardized as to the number of acetabular screws used during the procedure. Although all implants were initially stable, only one patient with 2 acetabular dome screws went onto failure. This was a retrospective review of radiographic data without patient reported outcomes or functional clinical outcome measures. Furthermore, the study did not have the statistical power to determine whether age, BMI, diagnosis, or sex were clinically significant. A long-term prospective, randomized clinical study comparing traditional ingrowth surfaces to newer, highly porous surfaces will be necessary to determine the clinical significance of the progression of radiolucencies observed on post-operative radiographs.

Another limitation to this study was the exclusion of femoral component data from the final analysis. The three reviewing surgeons did not specifically report on radiolucencies around the femoral component; however, analysis did include a notation for femoral subsidence. In this study, there were no femoral components that demonstrated any degree of subsidence.

\section{Conclusion}

The Trident Tritanium cup and its ultra-porous titanium biologic fixation surface exhibited a large number of patients with radiolucent lines, 96 out of 197 patients $(48.73 \%)$, around the prosthesis at 2 year follow-up. Further study with longer follow up will be required to ascertain the clinical significance of these radiolucencies and determine whether biologic fixation predictably occurs with this newer biologic surface. Our data does support the more recent studies showing a progression of the radiolucencies in some patients with Stryker Trident Tritanium acetabular components.

\section{References}

1. Naziri, Q., Issa, K., Pivec, R., Harwin, S., Delanois, R., \& Mont, M. (2013). Excellent Results of Primary THA Using a Highly Porous Titanium Cup. Orthopedics, 36:e390-4.

2. Hosny, H., El-Bakoury, A., Srinivasan, S., Yarlagadda, R., \& Keenan, J. (2018). Tritanium Acetabular Cup in Revision Hip Replacement: A Six to Ten Years of Follow-Up Study. J Arthroplasty, 33:2566-70.

3. Carli, A., Warth, L., de Mesy Bentley, K., \& Nestor, B. (2017 Feb). Short to Midterm Follow-Up of the Tritanium Primary Acetabular Component: A Cause for Concern. Journal of Arthroplasty, 32(2): 463-469.

4. Kaminski, P., Szmyd, J., Ambrozy, J., Jurek, W., \& Jaworski, J. (2016). Use of Trabecular Titanium Implants for Primary Hip Arthroplasty. Ortopedia Traumatologia Rehabilitacja, 18:461-70.

5. Sodhi, N., Izant, T., Diana, J., Del Gazio, D., Baratz, M., Levine, A., \& et al. (2018). Three-Year Outcomes of a Highly Porous Acetabular Shell in Primary Total Hip Arthroplasty. Orthopedics, 41:e154-7. 
6. Malahias, M.-A., Kostretzis, L., Greenberg, A., Nikolaou, V., Atrey, A., \& Sculco, P. (2020). Highly Porous Titanium Acetabular Components in Primary and Revision Total Hip Arthroplasty: A Systemic Review. Journal of Arthroplasty, 35:17371749 .

7. Perticarini, L., Zanon, G., Rossi, S., \& Benazzo, F. (2015). Clinical and Readiographic Outcomes of a Trabecular Titanium Acetabular Component in Hip Arthroplasty: Results at Minimum 5 Years Follow-Up. BMC Muskuloskelet Disord, 16:1-6.

8. Yoshioka, S., Nakano, S., Kinoshita, Y., Nakamura, M., Goto, T., Hamada, D., \& et al. (2018). Comparison of a Highly Porous Titanium Cup (Tritanium) and a Conventional Hydroxyapatite-Coated Porous Titanium Cup: A Retrospective Analysis of Clinical and Radiological Outcomes in Hip Arthroplasty Among Japanese Patients. J Orthopaedic Sci, 23:967-72.

9. Long, W., Nayyar, S., Chen, K., Novikov, D., Davidovitch, R., \& Vigdorchik, J. (2018). Early Aseptic Loosening of the Tritanium Primary Acetabular Component with Screw Fixation. Arthroplasty Today, 4:169-174.

10. Massari, L., Bistolfi, A., Grillo, P., Borre, A., Gigliofiorito, G., Pari, C., \& et al. (2017). Periacetabular Bone Densitometry After Total Hip Arthroplasty with Highly Porous Titanium Cups: A 2-Year Follow-Up Prospective Study. HIP Int, 27:551-7.

11. Bobyn, J. (4th Ed., 2011). Next Generation Porous Metals for Biologic Fixation. AAOS: Orthopaedic Knowledge Update Hip and Knee Reconstruction, 45.

12. Small, S., Berend, M., Howard, L., \& et al. (2013). High Initial Stability in Porous Titanium Acetabular Cups: A Biomechanical Study. J Arthroplasty, 28:510

13. Faizan, A. (2018). Clinical Evidence Behind Tritanium Shells. Stryker.

14. Muth, J., Poggie, M., Kulesha, G., \& et al. (2013). Novel Highly Porous Metal Technology in Artificial Hip and Knee Replacement: Processing Methadologies and CLinical Applications. J Minor Met Mater Soc, 65(2):318.

15. DeLee, J., \& Charnley, J. (1976). Radiological Demarcation of Cemented Sockets in Total Hip Replacement. Clin Orthop, 120:20.
16. National Joint Replacement Registry: Hip, Knee, and Shoulder Arthroplasty (2017). Australian Orthopaedic Association, 88-90.

17. National Joint Registry of England, Wales, Northern Ireland and Isles of Man (2017). 68-70.

18. Carli, A., Galmiche, R., Dobransky, J., \& Beaule, P. (2020 Sep). Radiographic assessment of the dynasty biofoam acetabular component with a minimum 2 years follow-up. Hip International, doi: 10.1177/1120700020958694.

\section{SUBMISSION HISTORY}

Submitted: December 10, 2020

Reviewed: February 21, 2021

Revised March 29, 2021

Accepted: April 4, 2021

Published: May 25, 2021

\section{AUTHOR AFFILIATIONS}

1 Dr David DeBoer MD; Dr Jeffrey Hodrick MD; Dr Matthew Christie Southern Joint Replacement Institute, 2400 Patterson St Suite 100, Nashville, TN (Direct inquires to David DeBoer, david@sjri.com)

\section{AUTHOR DISCLOSURES}

- DeBoer declares that research support was received from Stryker. Hodrick and Christie declare no conflict of interest in connection with this submission.

\section{COPYRIGHT \& OPEN ACCESS}

(C) 2021 DeBoer, Hodrick, Christie. All rights reserved. Authors retain copyright and grant the journal right of first publication with the work. Reconstructive Review is an open access publication and follows the Creative Commons Attribution-NonCommercial CC BY-NC. This license allows anyone to download works, build upon the material, and share them with others for non-commercial purposes as long as they credit the senior author, Reconstructive Review, and the Joint Implant Surgery \& Research Foundation (JISRF). An example credit would be: "Courtesy of (senior author's name), Reconstructive Review, JISRF, Chagrin Falls, Ohio". 